\title{
VIABILIDADE TÉCNICA E ECONÔMICA DA SUBSTITUIÇÃO DE FONTES CONVENCIONAIS DE ENERGIA POR BIOGÁS EM ASSENTAMENTO RURAL DO ESTADO DE SÃO PAULO ${ }^{1}$
}

\author{
MAURA S. T. ESPERANCINI ${ }^{2}$, FERNANDO COLEN ${ }^{3}$, OSMAR DE C. BUENO $^{4}$, \\ ANDRÉA E. B. PIMENTEL ${ }^{5}$, ELIAS J. SIMON ${ }^{6}$
}

\begin{abstract}
RESUMO: O objetivo deste estudo foi avaliar a viabilidade econômica da implantação de dois biodigestores com uso de dejetos animais em área do Assentamento de Trabalhadores Rurais no município de Itaberá - SP, no ano de 2005; um deles para o fornecimento de energia para os domicílios e outro para as atividades produtivas. Foram avaliados os benefícios referentes ao fornecimento de energia elétrica e térmica, a partir do biogás, para cinco domicílios da agrovila do assentamento e para as atividades produtivas, comparativamente aos custos de construção e operação para produção de biogás. Os resultados mostraram a viabilidade econômica da produção de gás em ambos os biodigestores. Foram gerados benefícios no valor de R $\$ 3.698,00$ por ano e $\mathrm{R} \$ 9.080,57$ por ano, nos biodigestores para os domicílios e produção, respectivamente; bem como o equivalente a $\mathrm{R} \$ 1.478,28$ por ano referentes à produção de biofertilizante. $\mathrm{O}$ custo anual do processo é de $\mathrm{R} \$ 1.218,50$ em cada biodigestor. O prazo de recuperação do investimento é de 2,5 anos e 11 meses, para a produção de biogás nos domicílios e na produção, respectivamente. Os resultados podem ser utilizados para subsidiar políticas públicas direcionadas ao aproveitamento de biomassa para a produção de energia a baixos custos no segmento da agricultura familiar.
\end{abstract}

PALAVRAS-CHAVE: biodigestores, economia de energia, assentamentos rurais.

\section{ECONOMICAL AND TECHNICAL FEASIBILITY OF THE CONVENTIONAL ENERGY SOURCE SUBSTITUTION BY BIOGAS IN RURAL SETTLEMENT OF SÃO PAULO STATE, BRAZIL}

\begin{abstract}
The aim of this work was to evaluate the economic feasibility of two biodigestion systems using animal excrements in a rural settlement, in Itaberá - SP, Brazil, in the year of 2005. One of them was constructed to supply biogas for five residences. The other one was constructed to supply biogas for production activities. The generated benefits related to the supply of electric and thermal energy were evaluated, using biogas for five residences and for production activities, in comparison to the annual costs of the biodigestion system construction and operation. The results showed economic feasibility of the biogas production in both cases. It was generated economic benefits of US\$ $1,766.84$ and US\$ 4,338.54 per year for the residential biodigestion system and production activities biodigestion system, respectively, as well as US\$706.20 per year for biofertilizer production. The costs were estimated in US\$ 582.18 per year in each biodigestion system. The pay-back was evaluated in 2.5 years for the residential case and 11 months for the production case, respectively. These results can be used to formulate public policies directed to biomass exploitation to produce low cost energy focused on familiar agriculture.
\end{abstract}

KEYWORDS: biodigestion systems, energy economics, rural settlements.

\footnotetext{
${ }^{1}$ Projeto financiado pelo CNPq.

${ }^{2}$ Eng a Agrônoma, Profa. Doutora, Departamento de Gestão e Tecnologia Agroindustrial, UNESP, Botucatu - SP, Fone: (0XX14) 3811-7164 - R: 31, maura@fca.unesp.br

${ }^{3}$ Engo Agrícola, Professor, Faculdades Sudoeste Paulista, Avaré - SP.

${ }^{4}$ Eng $^{\mathrm{o}}$ Agrônomo, Prof. Doutor, Departamento de Gestão e Tecnologia Agroindustrial, UNESP, Botucatu - SP.

${ }^{5}$ Economista, Profa. Doutora, Departamento de Gestão e Tecnologia Agroindustrial, UNESP, Botucatu - SP.

${ }^{6}$ Eng ${ }^{o}$ Agrônomo, Prof. Adjunto, Departamento de Gestão e Tecnologia Agroindustrial, UNESP, Botucatu - SP.

Recebido pelo Conselho Editorial em: 19-5-2006
}

Aprovado pelo Conselho Editorial em: 15-2-2007 


\section{INTRODUÇÃO}

Segundo dados do Balanço Energético Nacional referente ao ano de 2004, as principais fontes de energia para o consumo no segmento agropecuário foram óleo diesel (58\%), lenha (26\%), energia elétrica (15\%) e outros (1\%) (BRASIL, 2005). No triênio 2002-2004, dados oficiais disponíveis mostraram elevação dos preços pagos pela energia, pois os preços do óleo diesel apresentaram aumento de $41 \%$, da lenha aumento de $52 \%$ e da energia elétrica aumento de $36 \%$, em média, nesse período.

Os impactos da elevação do custo de energia fazem-se sentir com maior intensidade no setor rural de mais baixa renda, em geral, menos capitalizado e com menores condições de arcar com essa elevação de custos, tanto no que diz respeito ao consumo doméstico quanto para as atividades de produção.

Nesse sentido, o desenvolvimento e a implementação de alternativas tecnológicas com vistas à geração de energia a custos reduzidos para esse segmento podem gerar impactos socioeconômicos positivos. Uma das alternativas tecnológicas mais promissoras diz respeito ao aproveitamento da biomassa para geração de energia, que propicia uso mais racional dos recursos disponíveis na exploração agrícola, reduz a transferência de renda para outros agentes e diminui a dependência de fontes externas de energia.

Existem hoje diversas alternativas tecnológicas de aproveitamento da biomassa para geração de energia, tecnicamente viáveis para a agricultura familiar. Uma das alternativas que vem despertando grande interesse, é a tecnologia de biodigestão anaeróbia de resíduos dos animais, e particularmente de resíduos gerados com a criação animal, pela implantação de biodigestores.

Conforme apontado pela literatura, a tecnologia de biodigestão anaeróbia de dejetos animais, principalmente de suínos, apresenta diversas vantagens. Uma delas é a produção de biogás e biofertilizantes, produtos de elevado valor agregado, redução da poluição dos recursos hídricos, facilidade de implantação e operação, e redução da pressão sobre as matas pelo consumo de lenha (GASPAR, 2003). De outro lado, verifica-se que são ainda escassos os estudos com ênfase nos aspectos econômicos da implantação de biodigestores em pequenas escalas de produção, embora essa tecnologia seja encontrada em regiões com forte presença de atividade de suinocultura, em especial nos estados da região Sul do Brasil.

Assim, o objetivo deste estudo foi avaliar aspectos econômicos da implantação de dois biodigestores com o uso de dejetos animais na área III do Assentamento de Trabalhadores Rurais da Fazenda Pirituba II, município de Itaberá - SP, no ano de 2005, para a produção de biogás para consumo residencial e na produção de biofertilizante.

Considera-se que a avaliação de aspectos econômicos da implantação de biodigestores pode fornecer subsídios à formulação de políticas públicas voltadas ao desenvolvimento local mais sustentável de assentamentos do Estado de São Paulo, bem como fornecer subsídios à tomada de decisão quanto à implementação dessa tecnologia para o segmento da agricultura familiar de pequena e média escalas de produção animal, com vistas à produção mais sustentável.

\section{MATERIAL E MÉTODOS}

\section{Localização da área e características dos biodigestores}

Os biodigestores foram instalados na agrovila III da Fazenda Pirituba, localizada entre os municípios de Itapeva e Itaberá, a Sudoeste de São Paulo, composta de 27 lotes, com forma de exploração coletiva, feita por setores de produção: agricultura, pecuária e suinocultura; máquinas e implementos, apicultura, administração, construção e comércio.

Os biodigestores construídos são de modelo indiano e apresentam as seguintes dimensões, conforme o esquema a seguir: 


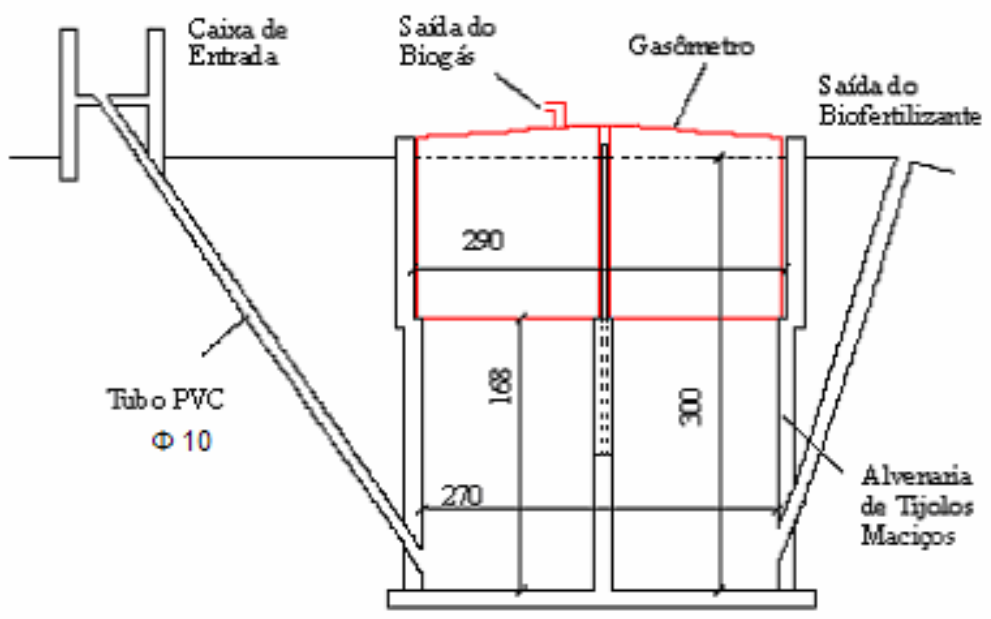

FIGURA 1. Dimensões dos biodigestores $(\mathrm{cm})$.

Foi utilizado esterco de suínos com $20 \%$ de sólidos totais. Para atingir a mistura de $8 \%$ de sólidos, foram utilizados $123 \mathrm{~kg}$ de esterco suíno e 184 litros de água, totalizando mistura de $307 \mathrm{~L}$, com Tempo de Retenção Hidráulica (TRH) de 50 dias, capacidade de produção de $0,35 \mathrm{~m}^{3}$ de biogás por $\mathrm{kg}$ de esterco, atingindo-se produção de $43 \mathrm{~m}^{3}$ de biogás por dia.

\section{Método}

Foram avaliados os benefícios referentes ao fornecimento de energia elétrica e térmica, a partir do biogás produzido pelo biodigestor, para cinco domicílios situados na agrovila III do assentamento, comparativamente aos custos de construção e operação do biodigestor. Foram avaliados os benefícios decorrentes do uso do biogás produzido em outro biodigestor para as atividades produtivas em substituição a outras fontes energéticas. Adicionalmente, avaliaram-se os benefícios decorrentes da produção de biofertilizantes. Os custos de implantação e de manutenção foram os mesmos para ambos os biodigestores.

Para determinar a viabilidade econômica do processo de biodigestão de dejetos bovinos e suínos para uso domiciliar, foram comparados dois indicadores: 1) Benefícios decorrentes do uso do biogás nos domicílios (BD) e na produção (BP) e da produção de biofertilizantes, e 2) Custo total de construção e operação do biodigestor (CT), ambos expressos em $\mathrm{R} \$$ ano $^{-1}$. Este método baseia-se na comparação entre os custos totais de produção e receitas obtidas, conforme MARTIN et al. (1998).

O valor dos benefícios não se refere às receitas econômicas, uma vez que o biogás não é vendido no mercado, mas sim à renda que se deixa de transferir para outros agentes (concessionárias de energia elétrica e distribuidoras de gás) ao reduzir o consumo de energia proveniente de fontes externas. Foram avaliados os benefícios da produção de biofertilizante com base na concentração e no valor de mercado dos nutrientes presentes.

Quanto ao biodigestor construído para o fornecimento de energia aos domicílios, definiu-se "a priori" que o biogás seria utilizado primordialmente para três funções: 1) fornecimento de gás para cocção; 2) aquecimento de água para banhos, e 3) iluminação interna. Dessa forma, os benefícios decorrentes da substituição de GLP e energia elétrica por biogás foram dados por:

$$
\mathrm{BD}=\mathrm{E}_{1}+\mathrm{E}_{2}+\mathrm{E}_{3}
$$

em que,

$\mathrm{BD}$ - benefícios decorrentes da economia de energia nas residências, $\mathrm{R} \$ \mathrm{ano}^{-1}$;

$\mathrm{E}_{1}$ - número de botijões de gás GLP que se deixa de adquirir ao longo de um ano x preço do botijão x número de domicílios atendidos, $\mathrm{R} \$ \mathrm{ano}^{-1}$; 
$\mathrm{E}_{2}$ - eliminação de gastos de energia de chuveiro elétrico em cada domicílio: tempo de uso diário por chuveiro (horas $\left.\mathrm{dia}^{-1}\right) \mathrm{x}$ consumo de energia por hora $(\mathrm{kWh}) \mathrm{x}$ preço de energia $\left(\mathrm{R} \$ \mathrm{kWh}^{-1}\right)$ x 365 dias ano $^{-1}$ x número de domicílios atendidos, $\mathrm{R} \$$ ano $^{-1}$, e

$\mathrm{E}_{3}$ - eliminação de gastos de energia em iluminação em cada domicílio: número de lâmpadas $\mathrm{x}$ tempo de uso diário (horas dia $\left.{ }^{-1}\right) \times$ consumo de energia por hora $(\mathrm{kWh}) \times$ preço de energia $\left(\mathrm{R} \$ \mathrm{kWh}^{-1}\right) \times 365$ dias ano $^{-1} \times$ número de domicílios atendidos, $\mathrm{R} \$ \mathrm{ano}^{-1}$.

A construção do biodigestor para a produção atende ao consumo de energia para três finalidades principais: 1) fornecimento de gás para lança-chamas para esfola de suínos após o abate; 2) fornecimento de energia para o triturador, e 3) aquecimento de água para um banheiro coletivo. Os benefícios decorrentes desse biodigestor foram dados por:

$$
\mathrm{BF}=\mathrm{F}_{1}+\mathrm{F}_{2}+\mathrm{F}_{3}
$$

em que,

$\mathrm{BP}$ - benefícios decorrentes da economia de energia nas atividades produtivas, $\mathrm{R} \$ \mathrm{ano}^{-1}$;

$\mathrm{F}_{1}$ - número de botijões de gás GLP que se deixa de adquirir ao longo de um ano para o lança-chamas $\mathrm{x}$ preço do botijão, $\mathrm{R} \$ \mathrm{ano}^{-1}$;

$\mathrm{F}_{2}$ - eliminação de gastos de energia do triturador: tempo de uso diário (horas $\operatorname{dia}^{-1}$ ) $\mathrm{x}$ consumo de energia hora $(\mathrm{kWh}) \times$ preço de energia $\left(\mathrm{R} \$ \mathrm{kWh}^{-1}\right)$ x 365 dias $^{\mathrm{ano}}{ }^{-1}$, $\mathrm{R} \$ \mathrm{ano}^{-1}, \mathrm{e}$

$\mathrm{F}_{3}$ - eliminação de gastos de energia em aquecimento de água para o banheiro coletivo: número de banhos $\mathrm{x}$ duração $\mathrm{x}$ consumo de energia pelo chuveiro $\mathrm{x}$ tarifa de energia elétrica x 365 dias.

Os custos de construção foram os mesmos para as duas unidades implantadas. São custos relativamente elevados e concentrados num único período, sendo rateados ao longo da vida útil dos biodigestores. Além dos custos de construção de biodigestores, foram determinados também os custos operacionais necessários para manter o equipamento em funcionamento; estes custos compõem-se predominantemente de mão-de-obra necessária para a coleta, manipulação e transporte de dejetos. Optou-se por considerar um custo de oportunidade para a mão-de-obra, uma vez que os trabalhadores responsáveis pela operação desenvolvem outras atividades produtivas no assentamento.

Não foi considerado o custo de oportunidade dos dejetos animais, matéria-prima da biodigestão, pois se considera que esses têm custo de oportunidade zero ou mesmo negativos, uma vez que usualmente são depositados a céu aberto, com efeitos poluentes e queda de bem-estar. Também não foram considerados os custos de manutenção do equipamento, uma vez que informações técnicas dão conta de que os custos de manutenção do biodigestor são não significativos.

Os custos de construção de um biodigestor foram agrupados em materiais, mão-de-obra de construção e horas-máquina de escavação. Os custos de condução de gás para os domicílios e adaptação dos equipamentos domésticos são pouco significativos e, em geral, o assentamento tem condições de realizá-los, mas foram considerados para efeitos de custos.

Na Tabela 1, mostra-se a quantidade de material necessário, serviços de escavação e mão-deobra de construção, especificando preço unitário, valor total e participação percentual no custo total de construção.

Como se pode verificar, o custo do gasômetro é o mais significativo, representando 51,62\% do custo do biodigestor, que totaliza $\mathrm{R} \$ 8.715,00$, tendo sido confeccionado em chapa de aço preta número 14 , com reforços de ferro chato de $3 / 4$ " x $1 / 8$ " e cantoneiras de $3 / 4$ " x 3/4" x $1 / 8$ "; com tubo-guia de aço galvanizado de 3". 
TABELA1. Custos de materiais para construção de um biodigestor em alvenaria, modelo indiano, 2005.

\begin{tabular}{llcrrr}
\hline Material & Unidade & Quantidade & Preço & Total & $\%$ \\
\hline Tijolo maciço comum & milheiro & 5,75 & 160,0 & 920,0 & 11,25 \\
Cimento & $\mathrm{saca}$ & 48,0 & 17,5 & 840,0 & 10,28 \\
Areia grossa & $\mathrm{m}^{3}$ & 1,0 & 88,0 & 88,0 & 1,08 \\
Areia fina & $\mathrm{m}^{3}$ & 3,0 & 48,0 & 168,0 & 2,06 \\
Brita 2 & $\mathrm{m}^{3}$ & 1,5 & 59,5 & 89,3 & 1,09 \\
Brita 1 & $\mathrm{m}^{3}$ & 0,5 & 59,5 & 29,8 & 0,36 \\
Impermeabilizante & 18 litros & 1,5 & 42,8 & 64,2 & 0,79 \\
Tubo PVC 150 mm & barra & 2,0 & 106,4 & 212,9 & 2,60 \\
Registro de esfera 50 mm & Unidade & 1,0 & 32,7 & 32,7 & 0,40 \\
Gasômetro & peça & 1,0 & $4.220,0$ & $4.220,0$ & 51,62 \\
Mangueira cristal (3/4") & m & 4,0 & 2,9 & 11,6 & 0,14 \\
Ferro galvanizado (3/8") & barra & 2,0 & 20,6 & 41,2 & 0,50 \\
Tubo galvanizado & Unidade & 0,5 & 246,6 & 123,3 & 1,51 \\
Junções & Unidade & 16,0 & 3,1 & 49,3 & 0,60 \\
Registro de esfera 32 mm & Unidade & 1,0 & 20,7 & 20,7 & 0,25 \\
Tubo PVC 32 mm & barra & 5,0 & 27,8 & 138,8 & 1,70 \\
Tubo PVC 50 mm & barra & 4,0 & 44,6 & 178,5 & 2,18 \\
Outros & & & & $7.260,0$ & 88,81 \\
\hline Total de materiais & & & & 150,0 & 1,84 \\
\hline Escavação & horas & 2,50 & 60,0 & 5,0 \\
\hline Mão-de-obra pedreiro & diárias & 20 & 27,00 & 540,0 & 6,61 \\
Mão-de-obra ajudante & diárias & 15 & 15,00 & 225,00 & 2,75 \\
\hline Total de serviços & & & 915,00 & 11,19 \\
\hline Total geral & & & & \\
\hline
\end{tabular}

Os custos anuais da implantação de biodigestores no assentamento foram os custos rateados do projeto de implantação pela vida útil e os custos operacionais de produção de biogás no ano. Os custos de implantação foram simplificados em depreciação e custo de oportunidade do investimento nos biodigestores. O método de depreciação utilizado foi o da depreciação linear, conforme NOGUEIRA (2001), dado por:

$$
\mathrm{D}=\frac{\mathrm{C}_{\mathrm{i}}-\mathrm{C}_{\mathrm{f}}}{\mathrm{Vu}}
$$

em que,

$\mathrm{D}$ - depreciação anual, $\mathrm{R} \$ \mathrm{ano}^{-1}$;

$\mathrm{C}_{\mathrm{i}}$ - custo de materiais depreciáveis;

$\mathrm{C}_{\mathrm{f}}$ - valor final do biodigestor, e

$\mathrm{Vu}$ - vida útil, anos.

O custo de oportunidade do capital foi dado em relação ao capital médio durante a vida útil e à taxa de juros de $12 \%$ ao ano (ASAE, 1999), expresso pela seguinte formulação:

$$
\mathrm{V}_{\mathrm{k}}=\frac{\mathrm{V}_{\mathrm{i}}-\mathrm{V}_{\mathrm{f}}}{2} \mathrm{r}
$$

em que,

$\mathrm{V}_{\mathrm{k}}$ - custo de oportunidade do capital, $\mathrm{R} \$ \mathrm{ano}^{-1}$;

$\mathrm{V}_{\mathrm{i}}$ - valor do capital total para a construção do biodigestor;

$\mathrm{V}_{\mathrm{f}}$ - valor final do biodigestor, $\mathrm{e}$

$r$ - taxa de juros anuais. 
Os custos operacionais foram dados pelo custo da mão-de-obra ocupada na coleta, transporte e abastecimento de resíduos dos biodigestores, determinado da seguinte forma:

$$
\mathrm{Co}=\mathrm{Fhh} \text { sal }
$$

em que,

Co - custos operacionais, $\mathrm{R} \$ \mathrm{ano}^{-1}$;

F - freqüência de abastecimento do biodigestor em número de vezes por ano;

hh-horas-homem dispendidas na coleta de dejetos, transporte e abastecimento dos biodigestores, e

sal - custo de oportunidade do trabalho, $\mathrm{R} \$$ hora de trabalho ${ }^{-1}$.

O custo total anual de produção do biogás foi dado por:

$$
\mathrm{CT}=\mathrm{D}+\mathrm{V}_{\mathrm{k}}+\mathrm{Co}\left(\mathrm{R} \$ \mathrm{ano}^{-1}\right)
$$

Os custos totais (CT), que são iguais para os dois biodigestores, foram comparados aos benefícios do uso do biogás nas residências (BD) e na produção (BP), e o valor do biofertilizante, produzido em cada biodigestor.

Utilizou-se, também, o indicador de tempo de recuperação do capital, dado pelo ano t, conforme NORONHA (1981):

$$
\sum_{\mathrm{t}=0}^{\mathrm{n}} \mathrm{L}_{\mathrm{t}}=0
$$

em que,

$\mathrm{L}_{\mathrm{t}}$ - fluxo de benefícios líquidos (benefícios menos custos), $\mathrm{R} \$ \mathrm{ano}^{-1}$;

$\mathrm{n}$ - vida útil do biodigestor, anos, e

$\mathrm{t}$ - contador de anos.

Os benefícios do biofertilizante foram determinados a partir da concentração de $\mathrm{N}, \mathrm{P}$ e $\mathrm{K}$ $\left(\mathrm{g} \mathrm{L}^{-1}\right)$, do volume de produção anual nos dois biodigestores e dos preços de mercado desses nutrientes.

\section{RESULTADOS E DISCUSSÃO}

Para o biogás com destino ao consumo doméstico, verificou-se que o biodigestor construído tem capacidade de fornecimento de energia contínua de $43 \mathrm{~m}^{3}$ por dia, suficiente para o abastecimento de cinco domicílios. Esses resultados foram obtidos a partir do dimensionamento do biodigestor, do consumo médio das diferentes fontes de energia nos domicílios analisados e uma carga composta de esterco suíno.

Um dos usos do biogás é dado pela substituição do gás GLP, adquirido para fins de cocção. Verificou-se que cada domicílio utiliza, em média, 1 botijão por mês, sendo utilizados 12 botijões ao ano. Ao custo de R\$30,00 por botijão, em cada domicílio, verifica-se economia de custos de $\mathrm{R} \$ 360,00$ por ano.

Outra utilização para o biogás é o aquecimento de água para banhos. Verifica-se que o chuveiro elétrico apresenta consumo de $5,40 \mathrm{kWh}$, sendo utilizadas 243,33 horas por ano, totalizando um consumo de $1.314,00 \mathrm{kWh}$ por ano em cada domicílio, com aquecimento de água para banho. A uma tarifa de energia elétrica estimada de $\mathrm{R} \$ 0,26$ por $\mathrm{kWh}$, para cada domicílio é gerada economia anual no uso do chuveiro de $\mathrm{R} \$ 341,64$ por ano.

Foi substituído um ponto de iluminação interno com uma lâmpada incandescente de 100 watts, utilizada durante quatro horas por dia. Nessas condições, o consumo anual é de $146,00 \mathrm{kWh}$. A uma tarifa de $\mathrm{R} \$ 0,26$ por $\mathrm{kWh}$, totaliza-se economia anual para cada domicílio de $\mathrm{R} \$ 37,96$. Esses dados, bem como a equivalência para cinco domicílios atendidos, em cada item de consumo, são apresentados na Tabela 2. 
TABELA 2. Benefícios decorrentes da economia de gastos pela substituição de energia elétrica e gás GLP por biogás, em assentamento rural em São Paulo, 2005.

\begin{tabular}{lccccc}
\hline Itens de consumo & $\begin{array}{c}\text { Consumo anual } \\
\text { um domicílio }\end{array}$ & $\begin{array}{c}\text { Consumo anual } \\
\text { cinco } \\
\text { domicílios }\end{array}$ & $\begin{array}{c}\text { Preço/ } \\
\text { unidade }\end{array}$ & $\begin{array}{c}\text { Total para um } \\
\text { domicílio } \\
\left(\mathrm{R} \$ \text { ano }^{-1}\right)\end{array}$ & $\begin{array}{c}\text { Total para cinco } \\
\text { domicílios } \\
\left(\mathrm{R} \$ \text { ano }^{-1}\right)\end{array}$ \\
\hline Botijões de gás & $12 \mathrm{un}$ & $60 \mathrm{un}$ & $30,00 / \mathrm{un}$ & 360,00 & $1.800,00$ \\
Aquecimento de água & $1.314 \mathrm{kWh}$ & $6.570 \mathrm{kWh}$ & $\mathrm{R} \$ 0,26 / \mathrm{kWh}$ & 341,64 & $1.708,20$ \\
Iluminação & $146 \mathrm{kWh}$ & $730 \mathrm{kWh}$ & $\mathrm{R} \$ 0,26 / \mathrm{kWh}$ & 37,96 & 189,80 \\
Total & - & - & - & 739,60 & $3.698,00$ \\
\hline
\end{tabular}

Quanto aos benefícios decorrentes da economia gerada pelo biodigestor para a produção, verificou-se que são utilizados 52 botijões de gás GLP durante um ano. Ao preço de R \$30,00 por unidade, a economia de custos decorrentes do uso do biogás em substituição ao GLP chega a $\mathrm{R} \$ 1.560,00$ ao ano.

O triturador usado no assentamento para moagem de cana-de-açúcar e forragens apresenta consumo de $7,5 \mathrm{kWh}$, sendo utilizado todos os dias durante 40 minutos, totalizando $1.825 \mathrm{kWh}$ durante o ano. À tarifa de $\mathrm{R} \$ 0,26$ por $\mathrm{kWh}$ de energia elétrica, verifica-se economia de custo de $\mathrm{R} \$ 474,50$ por ano.

Para o aquecimento de água do banheiro coletivo, foram considerados 27 banhos diários, que é o número de adultos que trabalham no campo, com duração de 10 minutos, para um chuveiro que consome $5,4 \mathrm{kWh}$. No ano, são gastos $8.869,50 \mathrm{kWh}$ à tarifa de $\mathrm{R} \$ 0,26$ por $\mathrm{kWh}$, totalizando $\mathrm{R} \$ 2.306,07$ de economia anual de energia.

Nessas condições, não é utilizado todo o potencial de produção do biogás, gerando excedente de gás que pode ser canalizado diretamente para outras atividades, como a cozinha coletiva (uma vez que se trata de Agrovila, a distribuição do gás pode ser feita diretamente, sem a necessidade de envasamento), verificando-se que é gerado excedente de 158 botijões de gás que correspondem ao benefício adicional de $\mathrm{R} \$ 4.740,00$ por ano.

TABELA 3. Economia de gastos pela substituição de energia elétrica e gás GLP por biogás, em assentamento rural do Estado de São Paulo.

\begin{tabular}{lccc}
\hline Itens de Consumo & Consumo Anual & Preço/Unidade & Total $\left(\mathrm{R} \$ \mathrm{ano}^{-1}\right)$ \\
\hline Lança-chamas & 52 botijões & $\mathrm{R} \$ 30,00 /$ botijão & $1.560,00$ \\
Aquecimento de água & $8.869,50 \mathrm{kWh}$ & $\mathrm{R} \$ 0,26 / \mathrm{kWh}$ & $2.306,07$ \\
Motor do triturador & $1.825,00 \mathrm{kWh}$ & $\mathrm{R} \$ 0,26 / \mathrm{kWh}$ & 474,50 \\
Excedente (em gás) & 158 botijões & $\mathrm{R} \$ 30,00 /$ botijão & $4.740,00$ \\
Total & & & $9.080,57$ \\
\hline
\end{tabular}

Verificou-se que o investimento necessário para a produção de $43 \mathrm{~m}^{3}$ diários é de $\mathrm{R} \$ 190,00$ por $\mathrm{m}^{3}$. Esse custo é relativamente elevado, comparando-se a biodigestores de maior porte (1.500 $\mathrm{m}^{3}$ diários), que chega a $\mathrm{R} \$ 80,00$ por $\mathrm{m}^{3}$ (LASLOWSKI, 2004), o que pode ser explicado pela redução de custos em função do aumento da escala.

O biofertilizante, a partir de análises realizadas, apresentou as seguintes concentrações de macronutrientes: nitrogênio $\left(1,60 \mathrm{~g} \mathrm{~L}^{-1}\right)$, fósforo $\left(0,52 \mathrm{~g} \mathrm{~L}^{-1}\right)$ e potássio $\left(0,45 \mathrm{~g} \mathrm{~L}^{-1}\right)$. Considerandose que são produzidos 184.720 litros de biofertilizante por ano, nos dois biodigestores, foram obtidos os resultados constantes da Tabela 4.

Verificou-se que a produção anual do biofertilizante para os dois biodigestores gera um valor adicional anual de $\mathrm{R} \$ 1.478,28$, sem descontar os custos de aplicação, que podem ser maiores que os custos da aplicação mineral. 
TABELA 4. Benefícios gerados pela produção de biofertilizantes, em dois biodigestores, ano 2005.

\begin{tabular}{lccc}
\hline Nutrientes & Produção Anual $(\mathrm{em} \mathrm{kg})$ & Preço de Mercado $\left(\mathrm{R} \$ \mathrm{~kg}^{-1}\right)$ & Receita Total $\left(\mathrm{R} \$ \mathrm{ano}^{-1}\right)$ \\
\hline Nitrogênio & 296,00 & 3,87 & $1.142,31$ \\
Fósforo & 96,00 & 3,22 & 309,51 \\
Potássio & 83,00 & 1,90 & 26,46 \\
Total & & & $1.478,28$ \\
\hline
\end{tabular}

Os materiais usados na construção do biodigestor foram considerados itens depreciáveis. Como a vida útil de um biodigestor de alvenaria é bastante longa, para fins de determinação do rateio dos custos de implantação ao longo do tempo, foi considerado o valor de 20 anos, conforme indicado por NOGUEIRA \& ZÜRN (2005). Dessa forma, a depreciação resulta em R\$363,00 anuais. Outro fluxo de custo considerado foi o de oportunidade do capital investido na construção do biodigestor, resultando em $\mathrm{R} \$ 490,50$ ao ano.

Os custos de operação dizem respeito ao trabalho necessário para manter o biodigestor em operação, ou seja, coleta, transporte e abastecimento do biodigestor. Esse trabalho é diário e exige duas pessoas que realizam essa operação em 30 minutos. Considerando-se o custo de oportunidade do trabalho, com base no salário médio por hora pago pela cooperativa, que foi de $\mathrm{R} \$ 1,00$, obteve-se o valor de R $\$ 365,00$ por ano. Portanto, o custo total do sistema é de R \$ 1.218,5 ao ano, considerando-se a depreciação, o custo de oportunidade do capital e os custos de operação (Tabela 5).

TABELA 5. Custos anuais de implantação e operação de um biodigestor em alvenaria, modelo indiano, 2005.

\begin{tabular}{lc}
\hline Itens de Custo & Valor $\left(\mathrm{R} \$\right.$ ano $\left.^{-1}\right)$ \\
\hline Depreciação & 363,00 \\
Juros sobre capital imobilizado & 490,50 \\
Mão-de-obra de operação & 365,00 \\
Total & $1.218,50$ \\
\hline
\end{tabular}

Os resultados indicam que os benefícios advindos do biogás para o uso domiciliar apresentaram vantagem comparativa em relação ao uso de fontes convencionais, como energia elétrica e GLP. As economias de energia do sistema resultaram em R \$3.698,00 por ano frente aos custos totais que foram de $\mathrm{R} \$ 1.218,50$ ao ano. Nessas condições, verificou-se que o equipamento utilizado para os domicílios pode ser amortizado num prazo de 2,5 anos. Também para o biodigestor direcionado à produção, os resultados mostram a viabilidade econômica do processo, pois frente ao mesmo custo anual, foram geradas economias de custos de $\mathrm{R} \$ 9.080,57$ por ano, o que permite que o investimento seja recuperado em 11 meses.

SOUZA et al. (2006), utilizando metodologia e tipo de investimentos diferentes (biodigestores, motor elétrico e conjunto de irrigação), totalizando $\mathrm{R} \$ 60$ mil, apresentaram período de retorno que varia entre 1,80 e 2,65 anos, dependendo do tempo de uso diário do gerador. Outro estudo, de JORDAN et al. (2004), mostra que um biodigestor com capacidade de produção de $150 \mathrm{~m}^{3}$ diários de biogás apresenta investimento inicial de $\mathrm{R} \$ 20$ mil e tempo de retorno de aproximadamente 15 meses.

A flexibilidade do equipamento quanto à destinação final da energia para biogás ou energia elétrica permite que sejam feitas economias de acordo com os preços de mercado do GLP ou da energia elétrica e dos custos adicionais de adaptação de equipamentos elétricos para utilizar o biogás na geração de eletricidade vis-à-vis com os custos de distribuição do biogás.

A avaliação da tecnologia por parte dos assentados foi positiva. É importante destacar que a construção do biodigestor da produção foi realizada pelos assentados a partir da capacitação de mão-de-obra local. 


\section{CONCLUSÕES}

Do ponto de vista estritamente econômico, os resultados apontam para a viabilidade da implantação de biodigestores no assentamento, pois o montante de benefícios é superior ao fluxo de custos anuais decorrentes da implantação do equipamento.

Os resultados apresentados podem ser utilizados na formulação de políticas públicas a fim de implementar assistência técnica para a utilização de biodigestores, bem como podem ser utilizados para subsidiar propostas de programas de financiamento de aproveitamento de biomassa para fins de produção de energia, voltados a esse segmento da agricultura, tendo em vista a economia de custos gerada no processo, bem como as inequívocas vantagens ambientais.

Os custos de implantação desse tipo de biodigestor podem constituir obstáculo à adoção dessa tecnologia, pois são assentamentos e agricultores com baixa capacidade de investimento. $\mathrm{O}$ item de maior custo, o gasômetro, deve ser objeto de pesquisa com vistas à redução de custos, pela utilização de outros materiais.

\section{REFERÊNCIAS}

ASAE. AMERICAN SOCIETY OF AGRICULTURAL ENGINEERS. Standart. St Joseph, 1999. 349 p. (ASAE EP496.2).

BRASIL. Ministério das Minas e Energia. Balanço Energético Nacional. 2005. Disponível em: <http://www.mme.gov.br>. Acesso em: 12 jan. 2006.

GASPAR, R.M.B.L. Utilização de biodigestores em pequenas e médias propriedades rurais com ênfase na agregação de valor: um estudo de caso na região de Toledo - PR. 2003. 106 f. Dissertação (Mestrado em Planejamento e Estratégia Organizacional) - Universidade Federal de Santa Catarina, Florianópolis, 2003.

JORDAN, R.A.; CORTEZ, L.A.B.; NEVES FILHO, L.C.; LUCAS JÚNIOR, J.L.; BALDASSIN JÚNIOR, R. Comparativo econômico do uso de uma bomba de calor para aquecimento e resfriamento de água em laticínios em relação ao aquecedor elétrico e o sistema de refrigeração convencional. In: CONGRESSO BRASILEIRO DE ENGENHARIA AGRÍCOLA, 33., 2004, São Pedro. Anais ... Jaboticabal: Associação Brasileira de Engenharia Agrícola, 2004. 1 CD-ROM.

LASLOWSKI, M. Avaliação ambiental e econômica do biogás, obtido através da biodigestão anaeróbia dos dejetos da suinocultura. 2004. 63 f. Monografia (Trabalho de Graduação em Agronomia) - Pontifícia Universidade Católica do Paraná, Curitiba, 2004.

MARTIN, N.B.; SERRA, R.; OLIVEIRA, M.D.M.O.; ÂNGELO, J.A.; OKAWA, H. Sistema integrado de custos agropecuários - Custagri. Informações Econômicas, São Paulo, v.28, n.1, p.728, 1998.

NOGUEIRA, C.E.C.; ZÜRN, H.H. Modelo de dimensionamento otimizado para sistemas energéticos renováveis em ambientes rurais. Engenharia Agrícola, Jaboticabal, v.25, n.2, p.341-8, 2005.

NOGUEIRA, E. Análise de investimentos In: BATALHA, M.O. Gestão Agroindustrial. 3.ed. São Paulo: Atlas, 2001. 388 p.

NORONHA, J. F. Projetos agropecuários. São Paulo: Atlas, 1981. 245 p.

SOUZA, S.N.M.; COLDEBELLA, A.; SOUZA, J.; KOEHLER, A.C. Viabilidade econômica de uso do biogás da bovinocultura para geração de eletricidade e irrigação. In: CONGRESSO BRASILEIRO DE ENGENHARIA AGRÍCOLA, 35., 2006, João Pessoa. Anais ... Jaboticabal: Associação Brasileira de Engenharia Agrícola, 2006. 1 CD-ROM. 